\title{
Corrigendum: $3^{\prime}$ UTR seed matches, but not overall identity, are associated with RNAi off-targets
}

Amanda Birmingham, Emily M Anderson, Angela Reynolds, Diane Ilsley-Tyree, Devin Leake, Yuriy Fedorov, Scott Baskerville, Elena Maksimova, Kathryn Robinson, Jon Karpilow, William S Marshall \& Anastasia Khvorova

Nat. Methods 3, 199-204 (2006).

In Figure 3 of the original article, the genes in panels a-c were incorrectly identified. The correct gene names are: (a) Ppyr/LUC siRNA A, (b) ALPPL2 and (c) Ppyr/LUC siRNA B.

The figure legend should read:

Figure 3 | Systematic single base pair-mismatch analysis of siRNA functionality. (a-c) Effects of single base pair mismatch in siRNAs targeting Ppyr/LUC siRNA A (a), ALPPL2 (b) and Ppyr/LUC siRNA B (c). Native forms of all three siRNAs induce > 90\% gene knockdown. Position 1 refers to the $5^{\prime}$-most position of the antisense strand. The top base represents the antisense mutation, and the bottom base represents the mismatched target site nucleotide. 'Mock', lipid-treated cells; ' + ', native duplex. Arrows point to examples of positions that have equivalent bases with at least one other siRNA in the test group and show differences in functionality when particular base substitutions are made. Experiments were performed in triplicate. Error bars, s.d. from the mean. (d) Bar graph of overall impact of mismatch identity on siRNA function. 